\title{
Transcription factors Aryl hydrocarbon receptor and TGF-inducible early gene are involved in an axis modulating immune response in mosquitoes
}

\author{
Aditi Kulkarni ${ }^{1}{ }^{*}$, Ashmita Pandey ${ }^{1}$, Patrick Trainor ${ }^{2}$, Samantha Carlisle $^{3}$, Wanqin $\mathrm{Yu}^{1}$, \\ Phanidhar Kukutla ${ }^{1}$, Jiannong $\mathrm{Xu}^{1}$
}

${ }^{1}$ Biology Department, New Mexico State University, Las Cruces, NM 88003, USA

${ }^{2}$ Economics, Applied Statistics \& International Business Department, New Mexico State University, Las Cruces, NM 88003, USA

${ }^{3}$ Department of Chemistry and Biochemistry, New Mexico State University, Las Cruces, NM 88003, USA

Corresponding author: Jiannong Xu, jxu@nmsu.edu

avkulkarni@utep.edu

ashmita@nmsu.edu

ptrainor@nmsu.edu

samcarli@nmsu.edu

ivyyu@nmsu.edu

phanidhar.kukutla@gmail.com

\section{*Current address:}

Aditi Kulkarni: Biology department, University of Texas, El Paso, TX 79968, USA 


\begin{abstract}
Immune homeostasis balances effective defense against pathogens with avoiding the adverse effect of immune overactivation. AhR is a ligand activated transcription factor that transduces chemical signals into transcription of a variety of target genes. In this study, we demonstrate that mosquito AhR and TIEG mediate a transcriptional axis modulating immune response negatively. When AhR was activated by diet delivery of agonist kynurenine, the anti-bacterial immunity was compromised with a reduced survival at $24 \mathrm{hr}$ post bacterial challenge. In contrast, when AhR was inhibited by antagonists, CH223191 or SR1, the immunity was enhanced with increased survival. The observed immune enhancement via $A h R$ antagonist was corroborated by $A h R$ gene silencing via RNAi, which resulted in increased survival upon the infection. Exploration of transcriptomes following AhR inactivation, either pharmacologically or genetically, highlighted a set of genes that are infection inducible and AhR dependent, including genes that may mediate immune suppressive functions. One of the genes, TIEG (TGF- $\beta$ inducible early gene), a member of the Krüppel -like factor family of transcription factors, was further studied. TIEG is required for AhR mediated immune suppression. Silencing TIEG increased the survival and reversed the immune suppression mediated by kynurenine activation of AhR. Moreover, the mosquito cohorts with $A h R$ or $T I E G$ knockdown demonstrated similar transcriptomic responses upon infection. There were co-expression patterns shared between the respective cohorts treated with AhR antagonist or AhR and TIEG silencing. In the naïve mosquitoes where the IMD pathway was overactivated by silencing the inhibitor gene Caspar, co-silencing Caspar with AhR or TIEG resulted in a reduced survival, indicating the AhR-TIEG axis is required to prevent the adverse effect of overactivation of IMD without an infection. Together, AhR and TIEG are involved in a transcriptional axis that is critical for maintaining immune homeostasis.
\end{abstract}

Keywords Anopheles gambiae, mosquito, AhR, Krüppel like factor, TIEG, innate immunity, immune homeostasis 


\section{Introduction}

The mosquitoes, like many insects, have evolved an efficient innate immune system consisting of Toll, IMD, JAK-STAT and RNAi pathways to defend against infection with bacteria, fungi, viruses and parasites [1-6]. To maintain immune homeostasis various regulatory circuits have evolved in insects to avoid deleterious effects of overaggressive immune responses and immunopathogenesis [7-9]. It has been well documented that intrinsic negative regulators Cactus [10], Caspar [11] and SOCS [12] for the Toll, IMD and Jak-STAT pathways, respectively, delicately tether the immune pathways. Besides, chemical defense mechanisms play important roles for homeostasis. Xenobiotic sensors are located at the interface with the chemicals originated from associated microbiota or environment. These sensors recognize ligands and initiate responses to coordinate context dependent xenobiotic metabolism and immune defenses [13]. The aryl hydrocarbon receptor (AhR) is a ligand-activated transcription factor, which was initially identified in the 1970 s as a xenobiotic sensor recognizing the compound 2,3,7,8tetrachlorodibenzo-p-dioxin (TCDD) in mice [14]. Through studies over the past 5 decades, it is now widely acknowledged that AhR can recognize various endogenous and exogenous ligands [15] and participate in multiple physiological processes including immune regulation as well as xenobiotic metabolism [15-19]. AhR is an ancient protein with an ortholog identified in the placozoan Trichoplax and conserved up to humans [18, 19]. According to the studies in the fruit fly Drosophila melanogaster, the nematode Caenorhabditis elegans and the cnidarian Nematostella vectensis, the ancestral functions of AhR are related to the development of sensory structures as well as neural systems (reviewed in [18]). These ancestral functions are likely mediated by endogenous ligands. During evolution, AhR has further acquired the capability to recognize foreign chemicals as an add-on function [18, 19]. In vertebrates, AhR has been recognized as a critical player in coordinating transcriptional circuits for immune regulation upon recognition of certain xenobiotics $[16,20,21]$. The connections between a xenobiotic sensor and immune regulation unveil the role of chemical sensing systems in transducing non-self signals into proper responses to execute effective immune defense while maintaining homeostasis. In contrast to the rich studies about diverse functions of AhR in vertebrates, very little is known about the AhR in insects except for the roles in development. Recently, AhR in fruit fly and nematode has been shown to be able to recognize indoles derived from symbiotic bacteria and lead to the transcription of a gene set contributing to healthy life span [22]. In this study, we dissect the role of AhR in mosquito immunity. Our data demonstrate that AhR and Krüppel like factor TIEG are connected to a transcriptional axis mediating immune suppression, suggesting that at insect level AhR has acquired the function to modulate innate immune responses.

\section{Materials and Methods}

\section{Mosquitoes}

Anopheles gambiae G3 strain was used for this study. The G3 mosquitoes were reared in an insectary with $28^{\circ} \mathrm{C}$ and $80 \%$ humidity, a light/dark cycling of 14:10 hours. Larvae were cultured in water pans with food (1:1 mix of ground pellet of cat food and brewer yeast), and adults were maintained with $10 \%$ sucrose sugar meal, and fed on mice to acquire blood to produce eggs.

\section{Phylogenetic inference using AhR protein sequences}


The AhR protein sequences of representative organisms from invertebrates to mammals (Table S1) were used for inferring phylogenetic relationship. The sequences were aligned using the MUSCLE algorithm. A phylogenetic tree was made by Neighbor-Joining method using JonesTaylor-Thornton (JTT) model, with 500 bootstrap replications. A similar tree topology was generated by Maximum Likelihood method using JTT model with 500 bootstrap replications. Only the NJ tree is shown in Fig. S1. The domains were visualized in Simple Module Architecture Research Toll (SMART, http://smart.embl-heidelberg.de/) , and the peptide sequences of domains bHLH, PAS A and PAS B were extracted and protein sequence identity between these organisms were compared.

\section{Pharmacological manipulation of AhR activity in mosquitoes}

AhR antagonists and agonists were used to manipulate AhR activity in mosquitoes. In each case, chemicals were fed to newly emerged adult mosquitoes, 50-60 females per cohort, in 10\% sucrose solution for three days and then challenged with Serratia fonticola S1 (see below). Kynurenine (Kyn) is an endogenous ligand of AhR [23]. Kyn is generated during the oxidation of tryptophan, a reaction catalyzed by tryptopan-2,3-dioxygenase (TDO) in mosquitoes [24]. Kyn at $30 \mu \mathrm{M}$ (Sigma-Aldrich, K8625) was used as agonist to enhance AhR activity. We used two AhR antagonists CH223191 at $90 \mu \mathrm{M}$ (Sigma-Aldrich, C8124) and Stem Regenin (SR1) at $3 \mu \mathrm{M}$ (Selleckchem, S2858). Additionally, we used 680C91 (20 $\mu \mathrm{M})$ (Sigma-Aldrich, SML0287), a TDO inhibitor, to reduce endogenous Kyn production. These concentrations of the chemicals were chosen empirically based on resultant phenotypes.

\section{Bacterial infection}

Bacterium Serratia fonticola S1 was isolated from a wild caught specimen of Aedes albopictus, collected in Florida in July 2015. Bacteria were transformed with a plasmid expressing GFP as described previously [25]. The bacteria were grown overnight in Luria Bertani broth containing ampicillin $(100 \mu \mathrm{g} / \mathrm{ml})$ at $29^{\circ} \mathrm{C}$. Bacterial culture was normalize to $\mathrm{OD}_{600 \mathrm{~nm}}=1$ and diluted 1000 times with sterile $\mathrm{H}_{2} \mathrm{O}$, which yielded approximately 1000 colony forming unit $(\mathrm{CFU}) / \mu 1$, and approximately $100 \mathrm{nl}$ of this bacterial solution was injected intrathoracically into the hemocoel of An. gambiae at day 3 after AhR activity was manipulated as described above. Survival at 24 hours post infection was used to assess the anti-bacterial immunity. The single thorax of dead and surviving mosquitoes was homogenized in $50 \mu 1$ sterile water, and $30 \mu 1$ homogenates were spread to a LB plate with Ampicillin and cultured at $28{ }^{\circ} \mathrm{C}$ overnight. The colonies on the plate were examined under UV light to visualize GFP tagged bacteria. In Serratia injected mosquitoes, GFP tagged bacteria were recovered, while in sterile water injected mosquitoes no GFP tagged bacteria were detected. The data were generated from three experimental replicates. For each treatment, the survival rates across replicates were not statistically different (Fisher's exact test, $\mathrm{P}>0.05$ ), therefore the replicate data were pooled to compare the survival between the treatment and vehicle control using a Chi square test.

\section{RT-PCR}

Mosquito RNA was extracted from 15 females using Trizol reagent (Invitrogen, Cat\# 15596026). Genomic DNA contamination was removed by DNaseI treatment using TURBO DNA-free Kit (Invitrogen, AM1906). cDNA synthesis was carried out using NEB ProtoScript II Reverse 
Transcriptase (NEB, M0368S). The cDNA was used as template for RT-PCR to determine transcript abundance for various genes. The primers used were present in Table S2. No reverse transcriptase (NRT) and no template control (NTC) served as negative controls.

\section{RNAi mediated gene knockdown in mosquitoes}

RNAi mediated AhR, TIEG and Caspar knockdowns were performed. For dsRNA preparation, a given target gene fragment was PCR amplified using gene specific primers with the T7 promoter sequence at the 5' end. The PCR products were used to synthesize dsRNA using a T7 RNA Polymerase Kit (Sigma-Aldrich RPOLT7-RO ROCHE). Generated dsRNAs were treated with TURBO DNA-free Kit (Invitrogen, AM1906) to remove DNA. The dsRNA of a GFP fragment was used as control dsRNA. For single gene silencing, $A h R$ and $T I E G, 0.5 \mu \mathrm{g} / \mu \mathrm{l}$ dsRNA was used for injection. To co-silence Caspar along with either $A h R$ or $T I E G$, respective dsRNA , each at $1.0 \mu \mathrm{g} / \mu \mathrm{l}$, were mixed for injection. Newly emerged An. gambiae female mosquitoes, 60 females per cohort, were subjected to intrathoracic injections with $\sim 100 \mathrm{nl}$ of dsRNA. Treated mosquitoes were maintained on $10 \%$ sucrose solution for three days. For bacterial challenge, cohorts of dsGFP control, dsAhR and dsTIEG were injected with Serratisa at day 4 post dsRNA treatment as described above. The gene knockdown efficacy was verified by RT-PCR with primers in Table S2.

\section{AhR Western blot}

Rabbit Polyclonal antibody against AhR was made at GenScript (New Jersey). The antigen was a peptide fragment in the PAS B domain of AhR protein (aa 208-394). For Western blot, mosquito proteins were extracted using Insect Cell-PE LB ${ }^{\mathrm{TM}}$ (G Biosciences, 786 $\square 411$ ) at three days post dsRNA injection. The protein samples $(20 \mu \mathrm{g} /$ well $)$ were separated by SDS-PAGE gel and transferred to a nitrocellulose membrane. The membrane was probed by the AhR antibody (1:1000). GAPDH antibody (GeneTex, GTX100118) was used as loading control. HRP labelled secondary antibody was used to detect signal using KPL LumiGLO kit following manufacturer's instruction. The blot was scanned to visualize the signal using the IMAGE STUDIO, Version 5.x, LI-COR.

\section{Transcriptome analysis}

RNA-sequencing (RNAseq) was used to compare transcriptomes between samples with AhR manipulation. To examine the effect of pharmacological AhR inhibition, three cohorts of females were used. Mosquitoes were fed with AhR antagonist SR1 or vehicle control for three days, and then were challenged with Serratia infection, as described above. An injury control (in which mosquitoes were injected with sterile $\mathrm{H}_{2} \mathrm{O}$ ) was included to control the effect of damage associated with injection. To examine the effect of gene knockdown, three cohorts of newly emerged females were used; each was treated with dsAhR, dsTieg, or dsGFP control, for 3 days, and then challenged with Serratia infection. Total RNA from 15 live mosquitoes at $24 \mathrm{hr}$ post challenge was isolated using Trizol reagent, and then DNaseI treatment was followed to remove DNA contamination. Triplicate RNA samples were prepared for each treatment. The RNA samples were further processed at Genewiz, NJ, where the cDNA libraries were prepared and sequenced using Illumina Hiseq, 2 x 150 bp paired-end chemistry. At least $25 \mathrm{M}$ clean reads were generated from each RNA sample, which provided a sequencing depth sufficient for transcriptome analysis. The reads were mapped against transcripts of An. gambiae (NCBI), 
implemented by using Array Star v.12 (DNAstar). Read counts were normalized using the median of ratios method [26] using DESeq2 software [27]. In determining normalized read counts, this method accounts for sequencing depth and RNA composition by calculating normalization factors for each sample in comparison to a pseudo-reference sample. After determining normalized read counts, an independent filter was utilized which removed transcripts with normalized counts less than 5. This resulted in a dataset of 10,714 transcripts. The clustering of all samples revealed that the replicate 2 of dsAhR/Serratia infection was not consistent with the other two replicates, likely due to a quality issue associated the replicate, therefore, this replicated was removed from analysis. Differentially expressed genes were identified using a negative binomial generalized linear model (GLM) available through DESeq2 [27]. Likelihood ratio tests were conducted to identify transcripts that exhibited differential expression between all groups. Pairwise differential expression comparisons were made and statistical significance was determined by computing q-values that preserve the False Discovery Rate (FDR) [28, 29] [26]. For example, concluding that a transcript was differentially expressed between two groups with a q-value of 0.05 would imply that there was a $5 \%$ chance (expected) that this conclusion was a false positive. To determine a lower dimensional representation of the transcriptomic data, principal components analysis (PCA) was conducted using regularized logtransformed (rlog) data. PCA seeks to find a small set of "principal components" that capture a large proportion of the variance in the original data [30]. The rlog data was determined using DESeq2, while the "prcomp" function in R [31] was utilized to determine the PCA. The proportion of the variance captured by each of the principal components was determined. Weighted gene co-expression network analysis (WGCNA) [32] was conducted to identify modules (or sets) of transcripts that are co-expressed. This analysis was conducted as follows. First, the topological overlap between transcripts in a signed and soft-thresholded correlation network determined from the rlog data was determined using the R package WGCNA [33]. From this, transcript modules were determined utilizing hierarchical clustering with an "average" link function and utilizing the hybrid version of the "Dynamic Tree Cut" algorithm [34]. To validate expression patterns revealed by RNAseq, a selected set of genes was measured using quantitative RT- PCR. Primers were presented in Table S2. The submission of the RNAseq reads to NCBI is in the process.

\section{Results}

\section{Manipulation of AhR activity affects immunity against bacterial infection in mosquitoes}

AhR is conserved from invertebrates to mammals. AhRs from mosquitoes An. gambiae and Aedes aegypti were clustered with the AhRs from fruit fly Drosophila melanogaster and nematode Caenorhabditis elegans in the same clade, while AhRs from zebrafish Danio rerio and mouse Mus musculus and human Homo sapiens were grouped together (Fig. S1A). The domains of bHLH and PAS A are involved in DNA binding and dimerization with cofactors, which are more conserved compared to PAS B that is involved in ligand binding (Fig. S1B). The higher level of divergence in PAS B suggests a diversity in ligand recognition. $A h R$ transcripts were detected in the whole body of mosquito larvae, pupae, and adults (Fig. S1 C), indicating that $A h R$ is constitutively expressed in all life forms of the mosquito. To investigate the immune regulatory role of $\mathrm{AhR}$, we pharmacologically manipulated $\mathrm{AhR}$ activity and then examined the survival of mosquitoes upon a bacterial infection. Kynurenine (Kyn) is known as an endogenous AhR ligand $[23,35]$, which is an intermediate metabolite of tryptophan oxidation catalyzed by 
tryptopan-2,3-dioxygenase (TDO) in mosquitoes [24]. TDO can be inhibited by 680C91[36, 37], which leads to the reduction of endogenous Kyn production. $\mathrm{CH} 233191[38,39]$ and SR1 [36] are known as AhR antagonists. After AhR activity was manipulated pharmacologically, the mosquitoes were challenged with bacteria by injecting Serratia into the hemocoel, and survival rate was recorded at $24 \mathrm{~h}$ post injection. As shown in Fig. 1A, the DMSO vehicle control mosquitoes with basal AhR activity had 58.6\% survival. When mosquitoes were fed with Kyn to boost AhR activity, the survival was reduced to 39.9\%. In contrast, when mosquitoes were fed with AhR antagonists CH233191, SR1 or 680C91, the survival was significantly increased to 78.7-85.4\%. Increased survival This phenotypic pattern was also observed in $A h R$ gene silencing using RNAi. When the $A h R$ gene was silenced by dsAhR via RNA interference, an increased survival rate $(82.4 \%)$ was observed in comparison with $57.8 \%$ of survival in the control mosquitoes treated with dsGFP (Fig.1B) mirroring results observed when AhR antagonists were given.

\section{Transcriptomic alterations upon AhR manipulation}

To identify genes that are regulated by AhR, we conducted RNAseq to interrogate An. gambiae transcriptomes. AhR activity was experimentally reduced by AhR antagonist SR1, and then the treated mosquitoes were challenged with Serratia infection. RNA samples were collected from surviving mosquitoes at $24 \mathrm{hr}$ post bacterial challenge and subjected to RNAseq. Transcriptomes were analyzed using normalized read counts, and genes that were differentially expressed were identified by DESeq2. In comparison of Serratia infection and injury control, the expression of 2102 genes was differentially altered (using a cutoff of q-values $<0.05$, Table S3), which we defined as infection responsive genes. Among these genes, 265 were upregulated and 145 were downregulated with at least 2-fold. Infection inducible gene set includes typical immune genes encoding such as immune pathway components (spaetzle1, spaetzle3, PGRPLB, Rel1 and Rel2), immune effectors (DEFs, CECs, TEPs and LRIMs, CLIP serine proteases, fibrinogens) and immune regulators (Serpins, suppressor of cytokine signaling (SOCS)). The AhR antagonist treatment affected the expression of 696 infection responsive genes. In comparison to infection only, the AhR antagonist resulted in 66 upregulated and 39 downregulated genes with at least 2fold differences. The major immune genes are not affected by the AhR antagonist, but potential immune suppressive genes, such as IAP4, SRPN6 and SRPN10 and SOCS require the presence of AhR for the response to infection. Expression patterns of Def1, Tep15, SRPN10 and TIEG were validated by qRT-PCR (Fig. S2).

\section{AhR - TIEG axis mediates a negative immune modulation}

One of the AhR dependent genes, AGAP009889, encodes a transcription factor in the Sp1like/Krüppel-like family [40], which is a ortholog of the vertebrate TGF- $\beta$-inducible earlyresponse gene (TIEG) [41]. The orthologous gene in Drosophila is Cabut, which is involved in development, metabolism and growth control [42-45]. In vertebrates, AhR and TGF- $\beta / S m a d$ signaling are connected to mediate immune suppression to maintain immune homeostasis [46], and TIEG is involved in TGF- $\beta /$ Smad signaling [47-49]. Based on this background information, we tested the hypothesis that TIEG is downstream of AhR, mediating the transcription of genes responsible for immune modulation. First, we examined the effect of TIEG silencing on the Serratia infection outcome in An. gambiae. As expected, silencing TIEG resulted in a survival $(90.1 \%)$, which was higher than that in the dsGFP control $(55.7 \%$, Chi-square, $\mathrm{P}<0.01)$ (Fig. 
Furthermore, we conducted RNAseq to compare the transcriptomes in response to the dsAhR and dsTIEG and AhR antagonist. Clustering analysis separated transcriptomes into two clusters (Fig. 2B). Injury control was separated from Serratia infection and antagonist/infection. In the other cluster, the dsAhR/infection and dsTIEG/infection were distinct from dsGFP. PCA analysis revealed that principal component 1 (PC1) and PC2 explained $61 \%$ and $11.2 \%$ of the variance, respectively, the transcriptome replicates of each treatment were closely clustered and different treatments separated their transcriptomes clearly (Fig. 3). Replicates of dsAhR and dsTIEG were located closely, indicating a similarity in their transcriptomic response. However, the replicates of dsGFP/Serratia infection were distinct from the replicates of Serratia infection, suggesting that the dsGFP treatment posed other impacts. In addition, there was an evident separation between AhR antagonist and gene silencing, which suggests that two approaches for AhR manipulation each may have impacts other than merely affecting the AhR activity. The AhR antagonist was administrated via diet, and gene silencing were achieved through injections, an invasive method. In mosquitoes, dsRNA triggers RNAi defense against virus [50], the dsGFP injection may have an unknown influence to the response to the following bacterial challenge. These confounding effects may contribute to the patterns revealed by PC1 and PC2. The PC3 and PC4 captured $7.3 \%$ and $4.1 \%$ of the variance, which may represent the effect of AhR manipulation more genuinely, the clusters of AhR antagonist, dsAhR and dsTIEG were closer and separated from the clusters of Serratia infection and dsGFP/infection (Fig. 3). This pattern evident in the PCA represented the transcriptomic responses that were driven by the AhR-TIEG signaling axis. This pattern was further supported by co-expression module analysis. The weighted gene correlation network analysis (WGCNA) was implemented to identify phenotype associated modules of co-expressed genes. WGCNA identified 35 modules from the entire dataset, each contains 23-1382 genes. The infection, AhR antagonist and AhR or TIEG silencing resulted in significant transcriptional alteration in 2102, 1631, 3285 and 3260 genes (defined by q-value $<0.05$, Table S2), respectively. There genes fall into different modules. There were 3 categories in which modules are affected by these treatments. The category I includes 9 modules that represent $1305 / 2102(62.1 \%)$ genes in the infection sample, the gene number in these modules were reduced to 498/1631 (30.5\%) genes in the AhR antagonist sample and $1007 / 3285(30.6 \%)$ genes in the dsAhR and 888/3260 (27.2\%) genes in the dsTIEG sample, suggesting that these genes in the 9 modules are AhR dependent. The category II includes 5 modules that includes $170 / 2102(8.1 \%)$ genes in the infection sample. These modules were enriched with more genes in the antagonist $(429 / 1631,26.3 \%)$ and the dsAhR $(1223 / 3285$, $37.2 \%)$ and the dsTIEG $(1185 / 3260,36.4 \%)$ sample. The category III represents 7 modules that were affected differently by the antagonist and gene silencing (Fig. S3). Together, the transcriptome comparisons indicate that the two manipulation approaches had resulted in broad impacts on transcriptomes, the PCA and WGCNA module analysis extracted effectively transcriptomic patterns caused by the AhR manipulation. The transcriptomic pattern was validated by qRT-PCR, the transcription of SRPN10, TEP15, SOCS was down regulated by AhR and TIEG silencing in response to the bacterial challenge (Fig. 2C). Next, we reasoned that the immune suppression resulting from AhR activation would be attenuated by TIEG silencing. Indeed, the survival reduction by Kyn feeding (demonstrated in Fig. 1 above) was by reversed by dsTIEG (Chi-square, $\mathrm{P}<0.01$, Fig. 2D). This result suggests that the TIEG acts downstream of the AhR. 


\section{AhR-TIEG axis maintains an immune balance when IMD pathway was overactivated}

From the observations above, we conclude that AhR and TIEG are involved in a signaling axis to control negative immune modulation. This axis may help to maintain immune homeostasis by preventing immune overactivation. To test this hypothesis, we created an over-activated immune state by silencing Caspar, the negative regulator of IMD signaling pathway [51], and examined the impact of $A h R$ or TIEG silencing on the life span in An. gambiae. As shown in Fig. 2E, the cohorts of dsGFP, dsAhR/dsGFP, dsTIEG/dsGFP and dsCaspar/dsGFP remained $~ 90 \%$ survival at day 3 post respective injections. However, when Caspar was co-silenced with either $A h R$ or $T I E G$, the survival was reduced to $70 \%$. The results show that the activation of IMD by depleting suppressor Caspar in the absence of an immune insult displays a normal survival only when AhR and TIEG are present, suggesting that the potential adverse effect of IMD over-activation is prevented by the AhR-TIEG signaling axis.

\section{Discussion}

AhR is conserved from invertebrate to vertebrate [18]. During evolution, in parallel to its ancestral role in development of sensory structure and neural systems, the AhR has diversified into a chemical sensor with binding affinity for broad intrinsic and/or extrinsic ligands derived from environment or associated microbiota [52]. This type of recognition transduces chemical signals into responses in various contexts, such as xenobiotic detoxification and immune regulation in vertebrates. At the invertebrate level, C. elegans and D. melanogaster have shown the capability of recognizing indoles from gut bacteria and mediating processes for a healthy life span [22]. In this study, we demonstrate the evidence that mosquito AhR and TIEG are involved in an axis of transcriptional cascade that participate in immune homeostasis. We used a bacterial infection model to examine the role of AhR function in regulating defense against the infection. When the AhR activity was activated by agonist Kyn, we observed a reduced survival upon bacterial challenge, which suggests that the anti-bacterial immunity was suppressed by AhR. On the contrary, when the AhR was inactivated by antagonists or TDO inhibitor, the immunity was enhanced, resulting in an increased survival upon the challenge (Fig. 1A). In line with the pharmacological manipulation, $A h R$ gene silencing increased mosquito survival upon the bacterial challenge (Fig. 1B). Next, AhR dependent genes in response to infection challenge were screened by RNAseq in mosquitoes in which AhR was inhibited by either antagonist or gene silencing (Table S3). The AhR manipulation did not affect transcriptionally the canonical immune effector genes, such as Defensin and Cecropin, suggesting that AhR pathway is functioning distinct from IMD and TOLL pathways. A set of genes were identified as bacterial challenge inducible and AhR dependent, which include genes with possible immune suppressive functions, such as SRPN10 and SOCS. This implied that AhR activation in the infection context may mediate transcriptional regulations of immune suppressive genes to maintain an immune balance. In line with this function, we further characterized the gene TIEG, encoding a $\mathrm{C} 2 \mathrm{H} 2$ transcription factor [42, 45], the homolog of Krüppel like factor, which is conserved from invertebrate to vertebrates [41]. TIEG has functional connections with TGF $\beta 1$ pathway in Drosophila and vertebrates [45, 49]. Recently, AhR-TGF $\beta 1 / \mathrm{Smad}$ signaling has been shown to mediate immune suppression in scurfy mice, a mouse autoimmune model [46]. We hypothesize that $\mathrm{AhR}$ and TIEG form a transcriptional axis to mediate immune regulation and supported this hypothesis with three lines of evidence. First, mosquitoes with silenced TIEG showed enhanced resistance to bacterial challenge with increased survival (Fig. 2A); second, the immune 
repressive effect induced by the AhR activation via Kyn was reversed by $A h R$ and TIEG silencing (Fig. 2D); third, silencing $A h R$ and silencing $T I E G$ resulted in similar transcriptional profiles by RNAseq (Fig. 3, Fig. S3). The immune suppressive function of AhR-TIEG axis is beneficial in maintain immune homeostasis. It has been shown that silencing IMD suppressor Caspar enhances mosquito immunity to fight against malaria parasites Plasmodium berghei and $P$. falciparum [11]. But in naïve conditions without an insult, immune machinery is leashed by negative regulators to prevent deleterious consequences, such as excessive inflammation, from happening [7]. The AhR-TIEG axis appears to act as a distinct immune regulatory loop that keeps the adverse effect of Caspar knockdown under control, as co-silencing Caspar and the $A h R-T I E G$ axis, the survival was compromised (Fig.2E).

Taken together, this study identified AhR and TIEG as components of a transcriptional axis which is involved in maintaining immune homeostasis in mosquitoes. Considering a recent finding that the AhR in nematode and fruit fly mediates healthy life span upon recognition of bacterial indole, it would be interesting to recognize and characterize indigenous and exogenous AhR ligands in mosquito immune contexts. Furthermore, to functionally elucidate the transcriptional axis, further work is required to characterize the role of TIEG in the cascade. Drosophila TIEG is the ancestor of Krüppel like factor 10 and 11 in the family of Sp1/Krüppellike zinc finger transcription factors [41]. Drosophila TIEG plays a role as a transcriptional repressor in S2 cells [42] and positively modulates TGF- $\beta$ signaling as well as JAK/STAT pathway in the patterning and cell proliferation during wing development [45]. The KLF10 and 11 in vertebrates mediate TGF- $\beta$ signaling [53]. TGF- $\beta$ signal pathway plays critical roles in immune regulations in both invertebrates and vertebrates [54-56]. There are cross talks between AhR and TGF- $\beta$ pathway in anti-inflammatory processes demonstrated in mammals [57-59]. A direct evidence of AhR/TGF- $\beta /$ Smad pathway in an anti-inflammation context was demonstrated lately in scurfy mice, a mouse model of immunodysregulation polyendocrinopathy enteropathy $X$-linked syndrome, in which ligand activated AhR-TGF- $\beta /$ Smad signaling mediates autoimmune suppression [40, 46]. The current findings that AhR signaling connects chemical sensing and immune regulation in mosquitoes warrant further study to elucidate the cross talk between xenobiotic defense and immune defense in insect level.

\section{Acknowledgments}

We thank Dr. Jennifer Curtiss in Biology Department at New Mexico University for her valuable suggestions along the study. We thank Dr. Michelle Riehle in Microbiology and Immunology at Medical College of Wisconsin for her constructive comments on the manuscript. This work was supported by the National Institutes of Health [SC1AI112786 to J.X.] and the National Science Foundation [No. 1633330 to J.X.]. The content is solely the responsibility of the authors and does not necessarily represent the official views of the National Institutes of Health.

\section{Author contributions}

Conceived and designed the study: JX and AK. Performed the experiments: AK, AP, WY, PK, JX. Analyzed the data: AK, JX, PT, SC. Wrote the paper: JX, AK. 


\section{Conflict of interest}

None declared.

\section{Figures}

A.

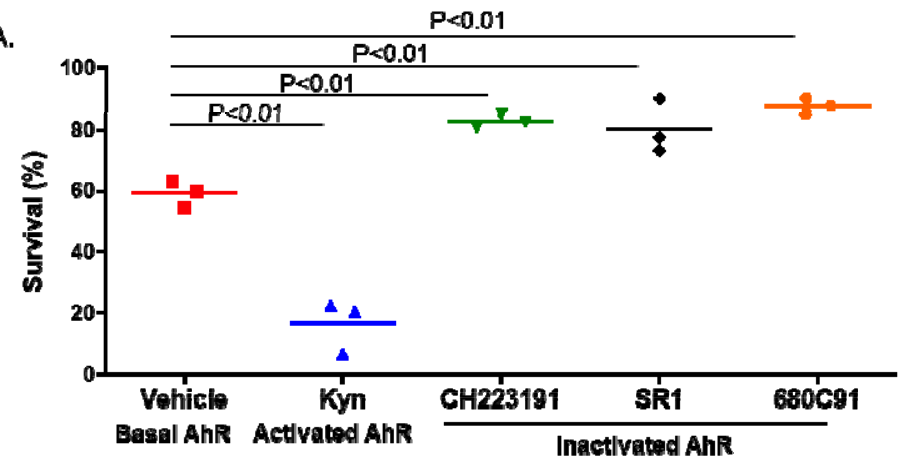

B.

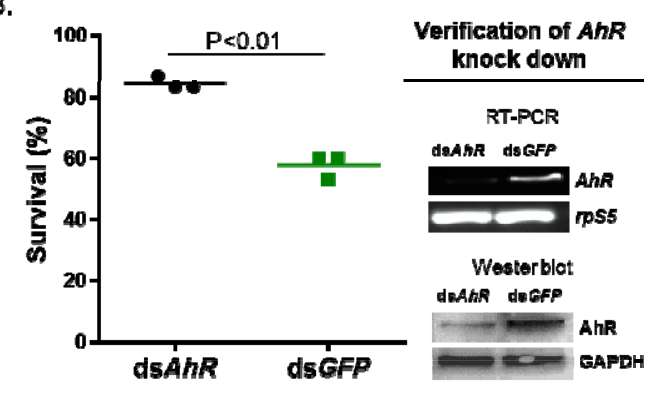

Figure 1. Mosquito survival upon bacterial challenge when AhR activity is manipulated pharmacologically or genetically. (A) DMSO vehicle control mosquitoes have with basal AhR activity; the AhR was activated by ligand Kyn-feeding. The AhR was inactivated by feeding AhR antagonists- (CH223191, SR1) or TDO inhibitor 680C91. The survival upon Serratia challenge was compared between the respective treatments. Data from three replicate experiments were presented. Each dot represents a cohort with 50 mosquitoes, average survival (\%) was denoted by the line. The survival difference was tested by the Chi square test. (B) $A h R$ gene was silenced by dsAhR, knockdown was verified by RT-PCR and Western blot. Mosquito survival upon Serratia challenge was compared between dsAhR and dsGFP control cohorts using the Chi square test. Data from three replicate experiments were presented. 
A.

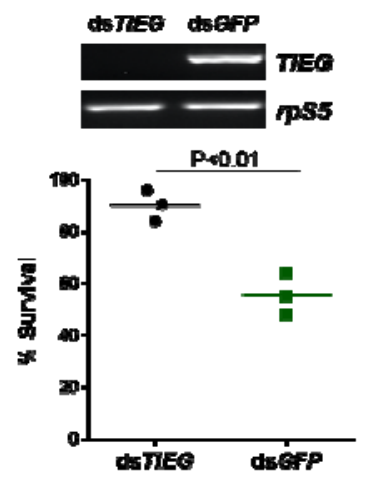

c.

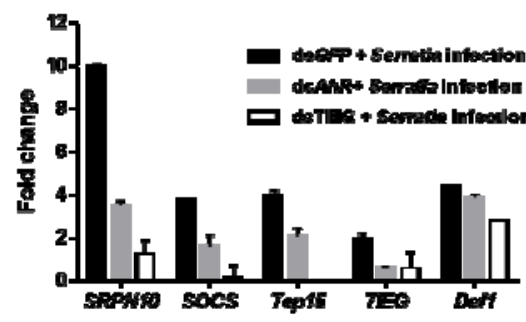

B.

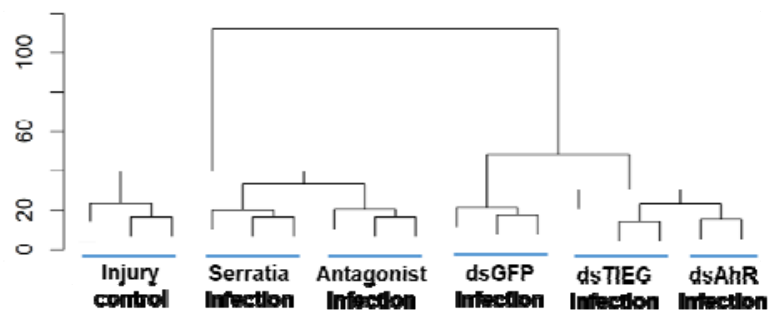

D.

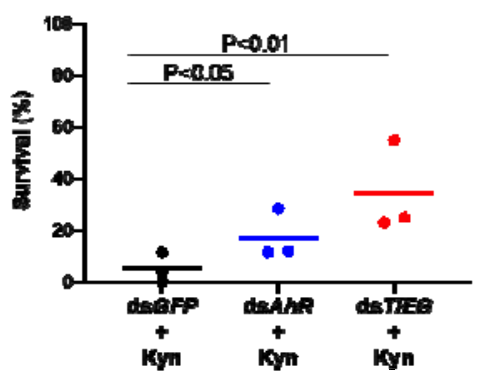

E

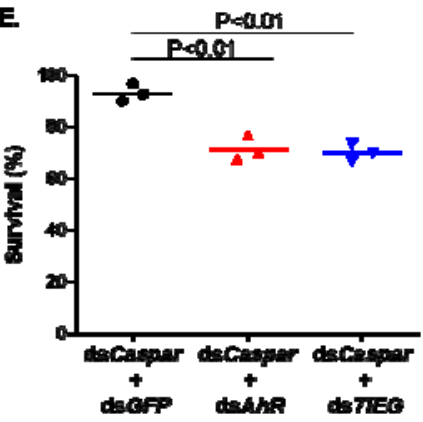

Figure 2. TIEG is mediating immunomodulation downstream of AhR. (A) The survival upon Serratia infection was higher in the dsTieg treated cohort than that in the dsGFP control, data were generated from three replicates (Chi square test, $\mathrm{P}<0.01$ ). The TIEG knockdown was verified by RT-PCR. (B) The transcriptomes of dsTIEG and dsAhR cohorts demonstrated a similar pattern in response to the Serratia challenge. The scale bar represents the distance between the clusters. (C) Validation by qPCR of selected AhR dependent and in genes in mosquitoes in which $A h R$ or TIEG was silenced. (D) The lower survival associated with Kyn mediated immune suppression was reversed by either $A h R$ or $T I E G$ silencing. Data were generated from three replicates. Each cohort had 30 mosquitoes. Newly emerged mosquitoes were injected with respective dsRNA for gene knockdown, and mosquitoes were maintained on $10 \%$ sugar with Kyn $(30 \mu \mathrm{M})$. The treated mosquitoes were challenged with Serratia at day 4 post knockdown. The survival was compared by the Chi square test. (E) The effect of dsAhR and dsTIEG on the survival of mosquitoes in which the IMD was overactivated by dsCaspar. Survival at day 3 post gene silencing was compared by the Chi square test. The data were generated from three replicates. Each cohort had 30 mosquitoes for the treatment. 

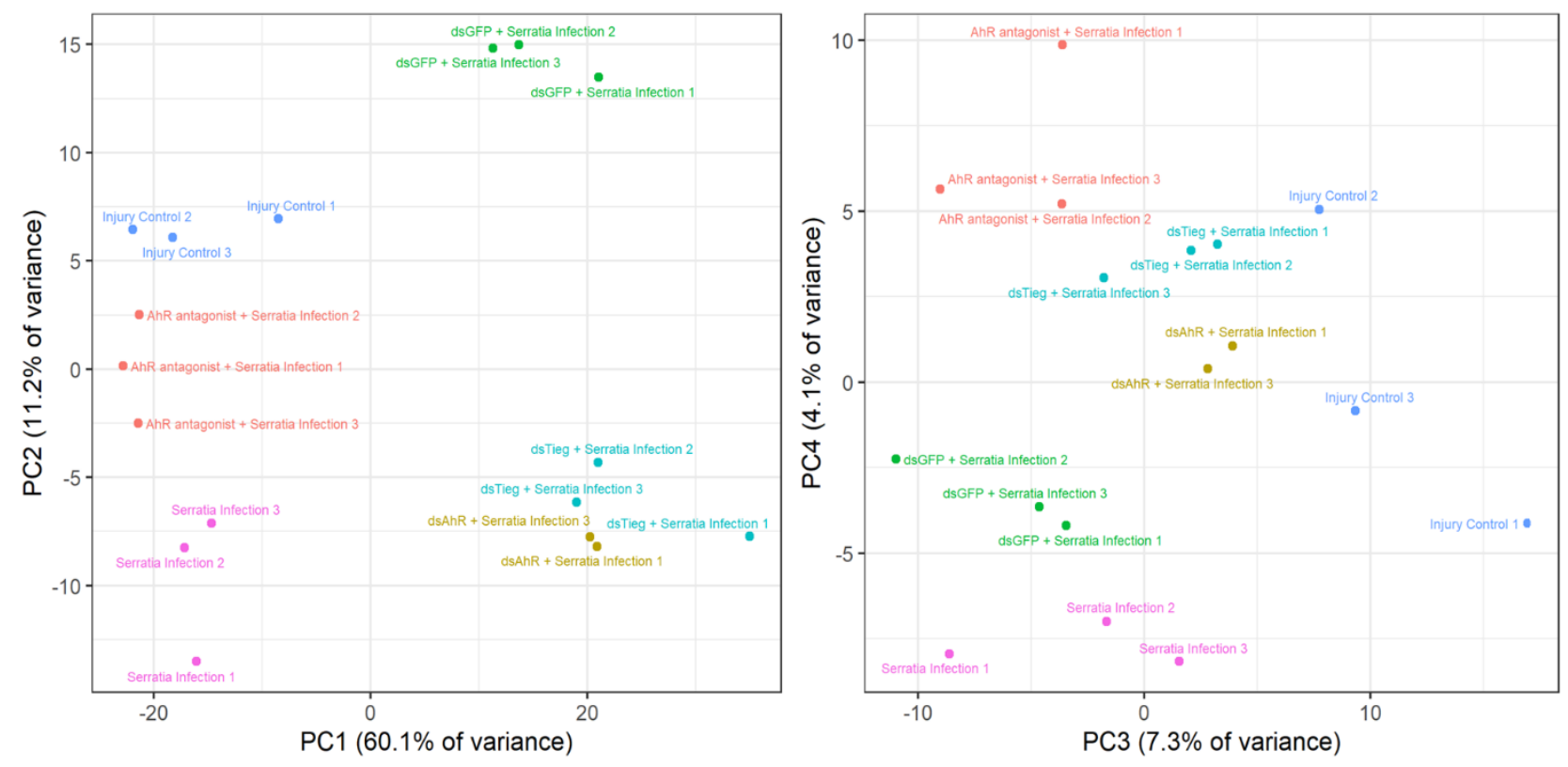

Figure 3. Principal component analysis (PCA) of transcriptomes. The PC1 and PC2 explained the major variance of transcriptomes with different treatments. The impact of AhR antagonist and dsAhR and dsTIEG on AhR activity in the infection context was better represented by PC 3 and PC4. See text for more interpretation. 


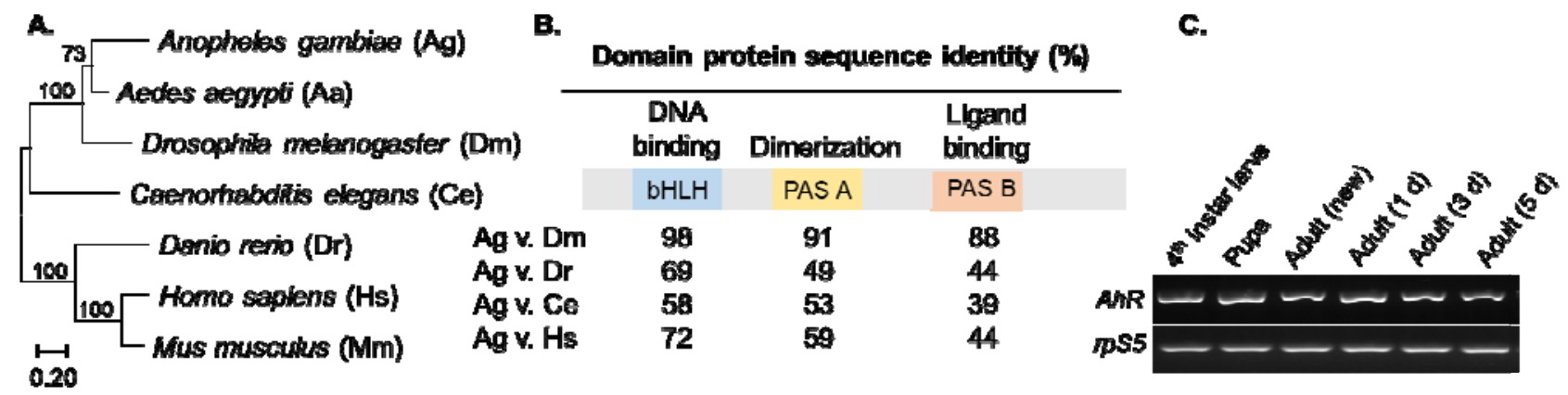

Figure S1. (A) The AhR phylogenetic tree inferred from amino acid sequence comparison using Neighbor-Joining method. Boostrap values were displayed on nodes. The scale bar indicates the genetic distance. (B) The level of conservation of domains bHLH, PAS A and PAS B, represented by identity of protein sequence. (C) $A h R$ is constitutively expressed in larvae, pupae and adults (newly emerged, 1-, 3-, 5-day old), assayed by RT-PCR, rpS5 was used as cDNA input control. 


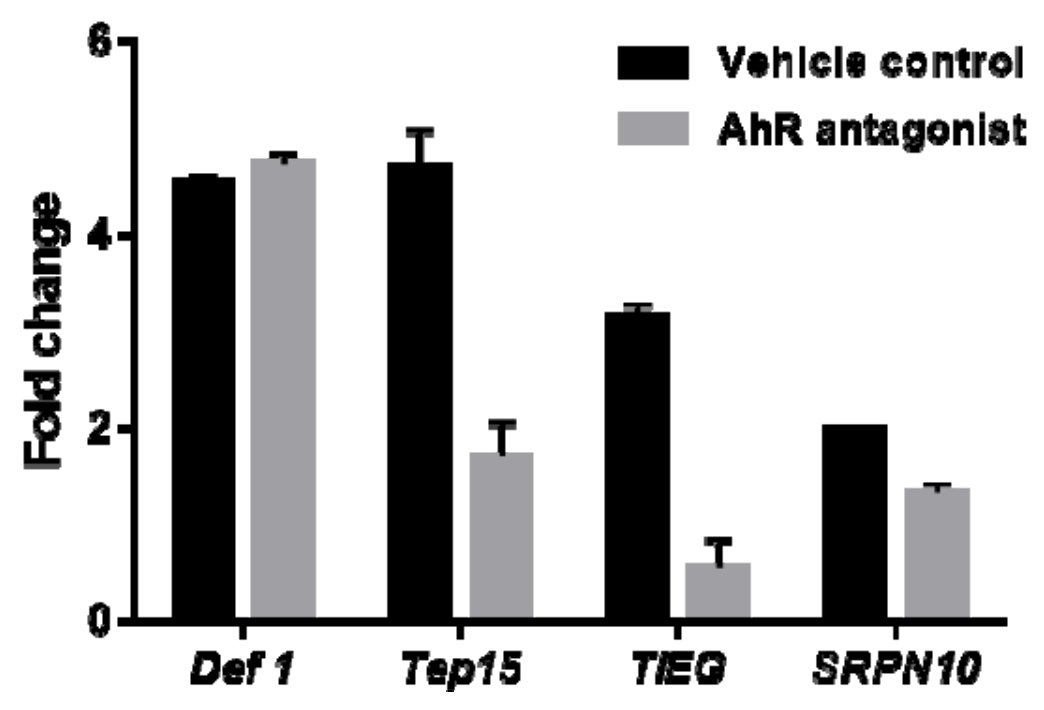

Figure S2. Transcriptional validation of selected genes by qRT-PCR. Selected genes that were identified in the transcriptome of the AhR antagonist/Serratia infection sample was chosen for the validation. Def1 represents a AhR independent gene, and Tep15 and TIEG and SRPN10 were AhR dependent genes. Fold change was calculated relative to injury control and the abundance of each transcript with respect to endogenous control rps5. Error bars represent SEM. 


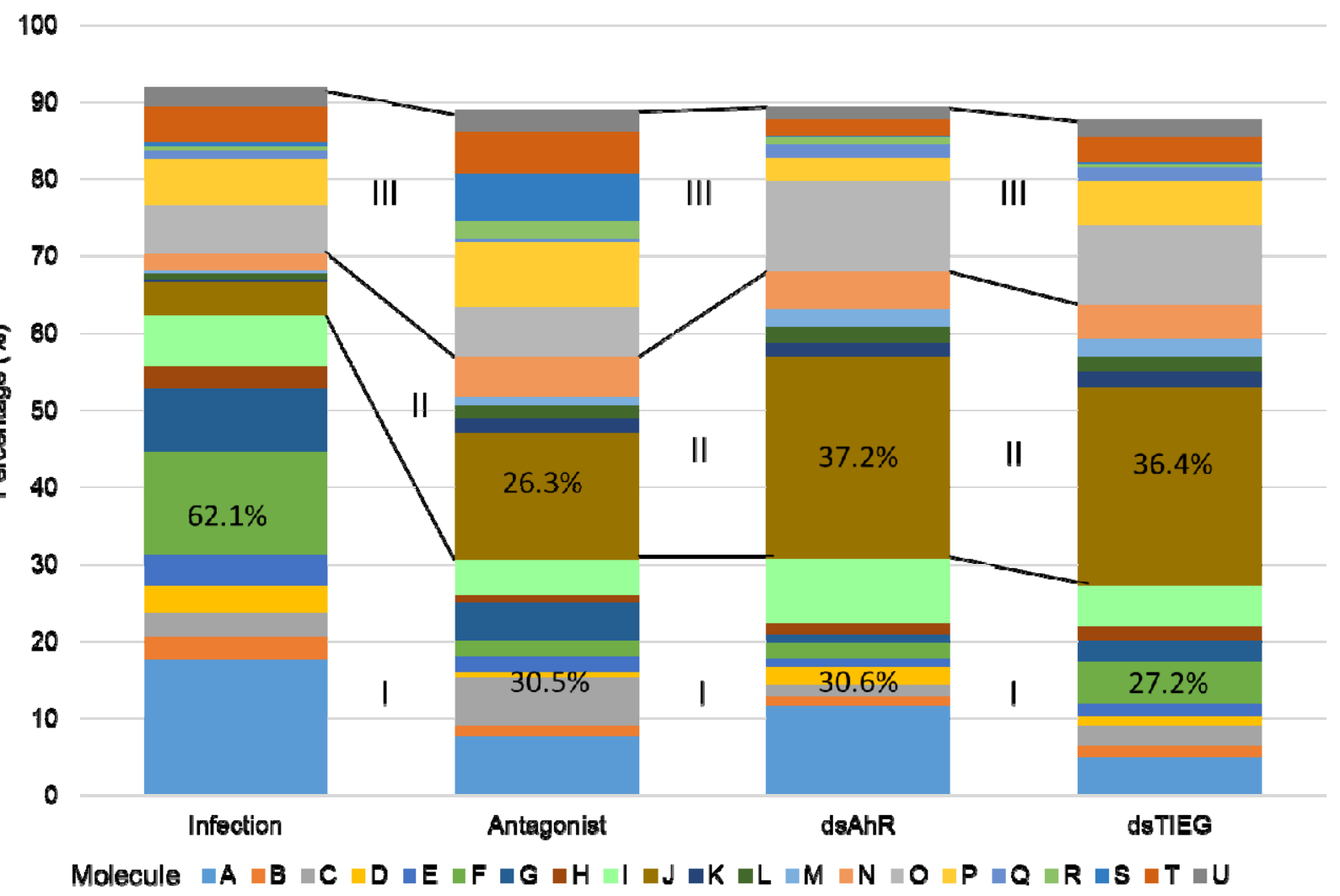

Figure S3. Co-expression modules analysis. The transcriptomic response in the cohorts of AhR antagonist, dsAhR and dsTIEG upon infection demonstrate similar impacts on co-expression modules. The number of genes in Category I modules was enriched in Infection cohort but was reduced to half in the AhR inactivated cohorts, while the number of genes in Category II modules was expanded in the AhR inactivated cohorts. The patterns represent the shared effects of AhR manipulation by both pharmacological and genetic approaches. 


\section{References}

1. Clayton AM, Dong Y, Dimopoulos G: The Anopheles innate immune system in the defense against malaria infection. J Innate Immun 2014, 6(2):169-181.

2. Kumar A, Srivastava P, Sirisena P, Dubey SK, Kumar R, Shrinet J, Sunil S: Mosquito Innate Immunity. Insects 2018, 9(3).

3. Lee WS, Webster JA, Madzokere ET, Stephenson EB, Herrero LJ: Mosquito antiviral defense mechanisms: a delicate balance between innate immunity and persistent viral infection. Parasit Vectors 2019, 12(1):165.

4. Merkling SH, Lambrechts L: Taking Insect Immunity to the Single-Cell Level. Trends Immunol 2020, 41(3):190-199.

5. Tawidian P, Rhodes VL, Michel K: Mosquito-fungus interactions and antifungal immunity. Insect Biochem Mol Biol 2019, 111:103182.

6. Bartholomay LC, Michel K: Mosquito Immunobiology: The Intersection of Vector Health and Vector Competence. Annu Rev Entomol 2018, 63:145-167.

7. Schneider DS: How and why does a fly turn its immune system off? PLoS Biol 2007, 5(9):e247.

8. Dionne MS, Schneider DS: Models of infectious diseases in the fruit fly Drosophila melanogaster. Dis Model Mech 2008, 1(1):43-49.

9. McKean KA, Yourth CP, Lazzaro BP, Clark AG: The evolutionary costs of immunological maintenance and deployment. BMC Evol Biol 2008, 8:76.

10. Riehle MM, Xu J, Lazzaro BP, Rottschaefer SM, Coulibaly B, Sacko M, Niare O, Morlais I, Traore SF, Vernick KD: Anopheles gambiae APL1 is a family of variable LRR proteins required for Rel1-mediated protection from the malaria parasite, Plasmodium berghei. PLoS One 2008, 3(11):e3672.

11. Garver LS, Dong Y, Dimopoulos G: Caspar controls resistance to Plasmodium falciparum in diverse anopheline species. PLoS Pathog 2009, 5(3):e1000335.

12. Gupta L, Molina-Cruz A, Kumar S, Rodrigues J, Dixit R, Zamora RE, Barillas-Mury C: The STAT pathway mediates late-phase immunity against Plasmodium in the mosquito Anopheles gambiae. Cell Host Microbe 2009, 5(5):498-507.

13. Mackowiak B, Hodge J, Stern S, Wang H: The Roles of Xenobiotic Receptors: Beyond Chemical Disposition. Drug Metab Dispos 2018, 46(9):1361-1371.

14. Poland A, Glover E, Kende AS: Stereospecific, high affinity binding of 2,3,7,8tetrachlorodibenzo-p-dioxin by hepatic cytosol. Evidence that the binding species is receptor for induction of aryl hydrocarbon hydroxylase. J Biol Chem 1976, 251(16):49364946.

15. Nebert DW: Aryl hydrocarbon receptor (AHR): "pioneer member" of the basichelix/loop/helix per-Arnt-sim (bHLH/PAS) family of "sensors" of foreign and endogenous signals. Prog Lipid Res 2017, 67:38-57.

16. Stockinger B, Di Meglio P, Gialitakis M, Duarte JH: The aryl hydrocarbon receptor: multitasking in the immune system. Annu Rev Immunol 2014, 32:403-432.

17. indoleFallarino F, Romani L, Puccetti P: AhR: far more than an environmental sensor. Cell Cycle 2014, 13(17):2645-2646.

18. Hahn ME, Karchner SI, Merson RR: Diversity as Opportunity: Insights from 600 Million Years of AHR Evolution. Curr Opin Toxicol 2017, 2:58-71.

19. Avilla MN, Malecki KMC, Hahn ME, Wilson RH, Bradfield CA: The Ah Receptor: Adaptive Metabolism, Ligand Diversity, and the Xenokine Model. Chem Res Toxicol 2020, 33(4):860879.

20. Gutierrez-Vazquez C, Quintana FJ: Regulation of the Immune Response by the Aryl Hydrocarbon Receptor. Immunity 2018, 48(1):19-33. 
21. Cella M, Colonna M: Aryl hydrocarbon receptor: Linking environment to immunity. Semin Immunol 2015, 27(5):310-314.

22. Sonowal R, Swimm A, Sahoo A, Luo L, Matsunaga Y, Wu Z, Bhingarde JA, Ejzak EA, Ranawade A, Qadota $\mathrm{H}$ et al: Indoles from commensal bacteria extend healthspan. Proc Natl Acad Sci U S A 2017, 114(36):E7506-E7515.

23. DiNatale BC, Murray IA, Schroeder JC, Flaveny CA, Lahoti TS, Laurenzana EM, Omiecinski $\mathrm{CJ}$, Perdew GH: Kynurenic acid is a potent endogenous aryl hydrocarbon receptor ligand that synergistically induces interleukin-6 in the presence of inflammatory signaling. Toxicol Sci 2010, 115(1):89-97.

24. Li JS, Han Q, Fang J, Rizzi M, James AA, Li J: Biochemical mechanisms leading to tryptophan 2,3-dioxygenase activation. Arch Insect Biochem Physiol 2007, 64(2):74-87.

25. Pei D, Jiang J, Yu W, Kukutla P, Uentillie A, Xu J: The waaL gene mutation compromised the inhabitation of Enterobacter sp. Ag1 in the mosquito gut environment. Parasit Vectors 2015, 8:437.

26. $\mathrm{Li} \mathrm{R}, \mathrm{Hu} \mathrm{K}$, Liu H, Green MR, Zhu LJ: OneStopRNAseq: A Web Application for Comprehensive and Efficient Analyses of RNA-Seq Data. Genes (Basel) 2020, 11(10).

27. Love MI, Huber W, Anders S: Moderated estimation of fold change and dispersion for RNAseq data with DESeq2. Genome Biol 2014, 15(12):550.

28. Storey JD, Tibshirani R: Statistical significance for genomewide studies. Proc Natl Acad Sci $U$ $S$ A 2003, 100(16):9440-9445.

29. Storey JD: The positive false discovery rate: a Bayesian interpretation and the q-value. Annals of Statistics 2003, 13(6):2013-2035.

30. Johnson RA: Applied Multivariate Statistical Analysis, 6th edn: Pearspm; 2019.

31. R: A language and environment for statistical computing. [https://www.R-project.org/]

32. Zhang B, Horvath $\mathrm{S}$ : A general framework for weighted gene co-expression network analysis. Stat Appl Genet Mol Biol 2005, 4:Article17.

33. Langfelder P, Horvath S: WGCNA: an R package for weighted correlation network analysis. BMC Bioinformatics 2008, 9:559.

34. Langfelder P, Zhang B, Horvath S: Defining clusters from a hierarchical cluster tree: the Dynamic Tree Cut package for R. Bioinformatics 2008, 24(5):719-720.

35. Nguyen LP, Bradfield CA: The search for endogenous activators of the aryl hydrocarbon receptor. Chem Res Toxicol 2008, 21(1):102-116.

36. Boitano AE, Wang J, Romeo R, Bouchez LC, Parker AE, Sutton SE, Walker JR, Flaveny CA, Perdew GH, Denison MS et al: Aryl hydrocarbon receptor antagonists promote the expansion of human hematopoietic stem cells. Science 2010, 329(5997):1345-1348.

37. Opitz CA, Litzenburger UM, Sahm F, Ott M, Tritschler I, Trump S, Schumacher T, Jestaedt L, Schrenk D, Weller $\mathrm{M}$ et al: An endogenous tumour-promoting ligand of the human aryl hydrocarbon receptor. Nature 2011, 478(7368):197-203.

38. Gerlach CV, Das SR, Volz DC, Bisson WH, Kolluri SK, Tanguay RL: Mono-substituted isopropylated triaryl phosphate, a major component of Firemaster 550, is an AHR agonist that exhibits AHR-independent cardiotoxicity in zebrafish. Aquat Toxicol 2014, 154:71-79.

39. Iida M, Bak SM, Murakami Y, Kim EY, Iwata H: Transient suppression of AHR activity in early red seabream embryos does not prevent the disruption of peripheral nerve projection by 2,3,7,8-tetrachlorodibenzo-p-dioxin. Aquat Toxicol 2014, 154:39-47.

40. Feinberg MW: Fine-tuning innate and adaptive immune responses: another KLFhanger. Focus on "Kruppel-like factor KLF10 regulates transforming growth factor receptor II expression and TGF-beta signaling in CD8+ T lymphocytes". Am J Physiol Cell Physiol 2015, 308(5):C359-361.

41. Munoz-Descalzo S, Belacortu Y, Paricio N: Identification and analysis of cabut orthologs in invertebrates and vertebrates. Dev Genes Evol 2007, 217(4):289-298. 
42. Belacortu Y, Weiss R, Kadener S, Paricio N: Transcriptional activity and nuclear localization of Cabut, the Drosophila ortholog of vertebrate TGF-beta-inducible early-response gene (TIEG) proteins. PLoS One 2012, 7(2):e32004.

43. Bartok O, Teesalu M, Ashwall-Fluss R, Pandey V, Hanan M, Rovenko BM, Poukkula M, Havula E, Moussaieff A, Vodala $\mathrm{S}$ et al: The transcription factor Cabut coordinates energy metabolism and the circadian clock in response to sugar sensing. EMBO $J$ 2015, 34(11):15381553.

44. Ruiz-Romero M, Blanco E, Paricio N, Serras F, Corominas M: Cabut/dTIEG associates with the transcription factor Yorkie for growth control. EMBO Rep 2015, 16(3):362-369.

45. Rodriguez I: Drosophila TIEG is a modulator of different signalling pathways involved in wing patterning and cell proliferation. PLoS One 2011, 6(4):e18418.

46. Cheng X, Haeberle S, Luca Shytaj I, Gama-Brambila RA, Theobald J, Ghafoory S, Wolker J, Basu U, Schmidt C, Timm A et al: NHC-gold compounds mediate immune suppression through induction of AHR-TGFbeta1 signalling in vitro and in scurfy mice. Commun Biol 2020, 3:10.

47. Subramaniam M, Hawse JR, Johnsen SA, Spelsberg TC: Role of TIEG1 in biological processes and disease states. $J$ Cell Biochem 2007, 102(3):539-548.

48. Papadakis KA, Krempski J, Reiter J, Svingen P, Xiong Y, Sarmento OF, Huseby A, Johnson AJ, Lomberk GA, Urrutia RA et al: Kruppel-like factor KLF10 regulates transforming growth factor receptor II expression and TGF-beta signaling in CD8+ T lymphocytes. Am J Physiol Cell Physiol 2015, 308(5):C362-371.

49. Johnsen SA, Subramaniam M, Janknecht R, Spelsberg TC: TGFbeta inducible early gene enhances TGFbeta/Smad-dependent transcriptional responses. Oncogene 2002, 21(37):57835790 .

50. Blair CD: Mosquito RNAi is the major innate immune pathway controlling arbovirus infection and transmission. Future Microbiol 2011, 6(3):265-277.

51. Kim M, Lee JH, Lee SY, Kim E, Chung J: Caspar, a suppressor of antibacterial immunity in Drosophila. Proc Natl Acad Sci U S A 2006, 103(44):16358-16363.

52. Kawajiri K, Fujii-Kuriyama Y: The aryl hydrocarbon receptor: a multifunctional chemical sensor for host defense and homeostatic maintenance. Exp Anim 2017, 66(2):75-89.

53. Spittau B, Krieglstein K: Klf10 and KIf11 as mediators of TGF-beta superfamily signaling. Cell Tissue Res 2012, 347(1):65-72.

54. Li MO, Wan YY, Sanjabi S, Robertson AK, Flavell RA: Transforming growth factor-beta regulation of immune responses. Annu Rev Immunol 2006, 24:99-146.

55. Sanjabi S, Oh SA, Li MO: Regulation of the Immune Response by TGF-beta: From Conception to Autoimmunity and Infection. Cold Spring Harb Perspect Biol 2017, 9(6).

56. Clark RI, Woodcock KJ, Geissmann F, Trouillet C, Dionne MS: Multiple TGF-beta superfamily signals modulate the adult Drosophila immune response. Curr Biol 2011, 21(19):1672-1677.

57. Monteleone I, Marafini I, Zorzi F, Di Fusco D, Dinallo V, Rizzo A, Sileri P, Sica G, Monteleone G: Smad7 Knockdown Restores Aryl Hydrocarbon Receptor-mediated Protective Signals in the Gut. J Crohns Colitis 2016, 10(6):670-677.

58. de Lima KA, Donate PB, Talbot J, Davoli-Ferreira M, Peres RS, Cunha TM, Alves-Filho JC, Cunha FQ: TGFbeta1 signaling sustains aryl hydrocarbon receptor (AHR) expression and restrains the pathogenic potential of TH17 cells by an AHR-independent mechanism. Cell Death Dis 2018, 9(11):1130.

59. Wei YL, Chen YQ, Gong H, Li N, Wu KQ, Hu W, Wang B, Liu KJ, Wen LZ, Xiao X et al: Fecal Microbiota Transplantation Ameliorates Experimentally Induced Colitis in Mice by Upregulating AhR. Front Microbiol 2018, 9:1921. 
bioRxiv preprint doi: https://doi.org/10.1101/2020.11.05.368985; this version posted November 5, 2020. The copyright holder for this preprint (which was not certified by peer review) is the author/funder. All rights reserved. No reuse allowed without permission. 
A.

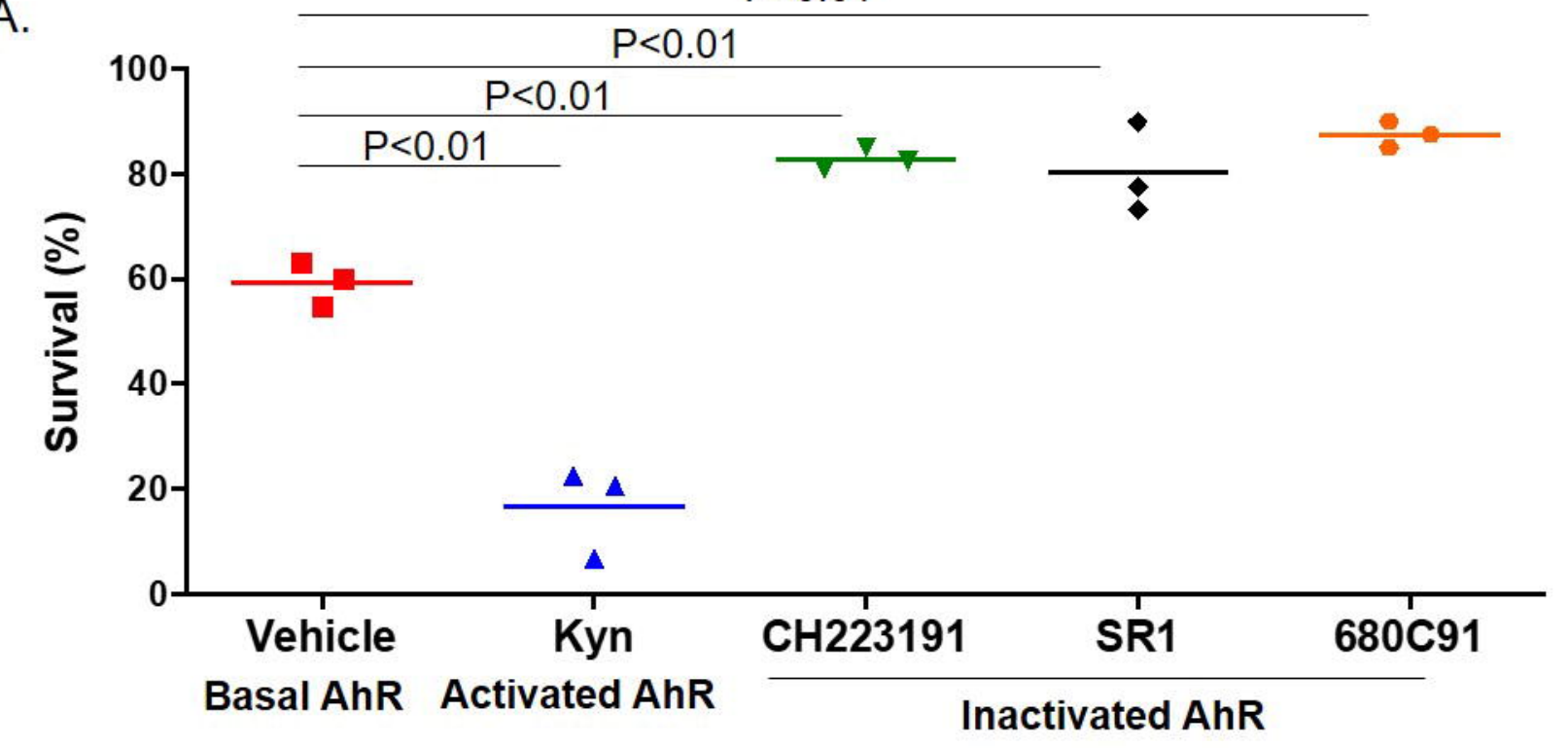

B.

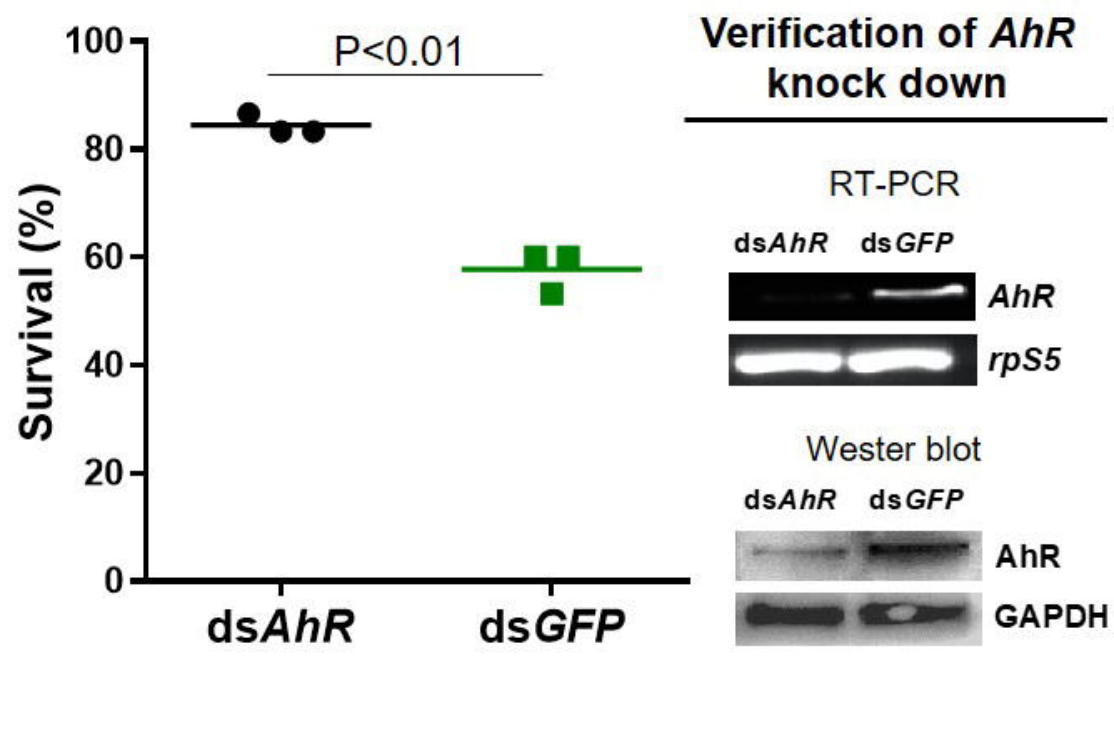


A.
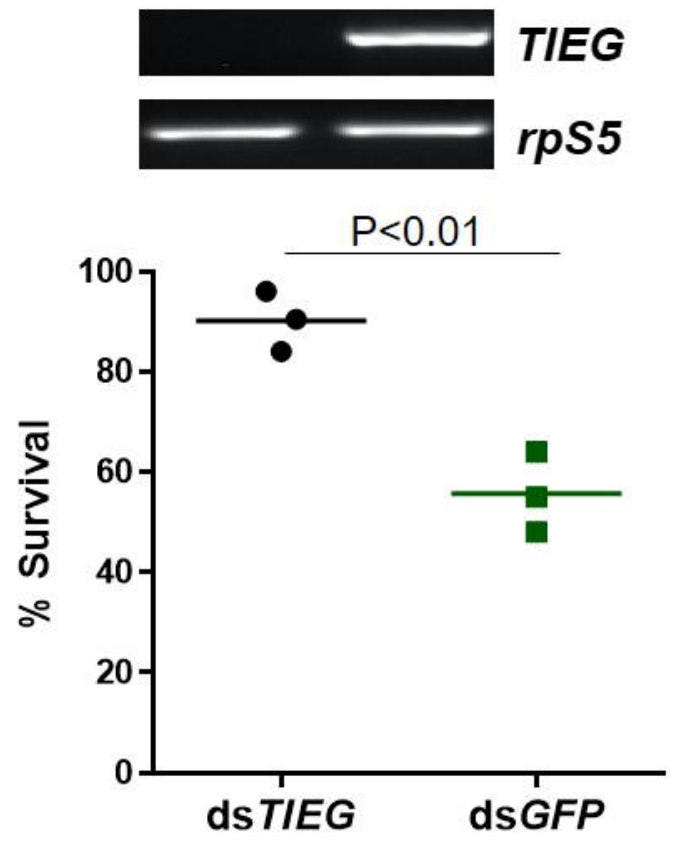

C.

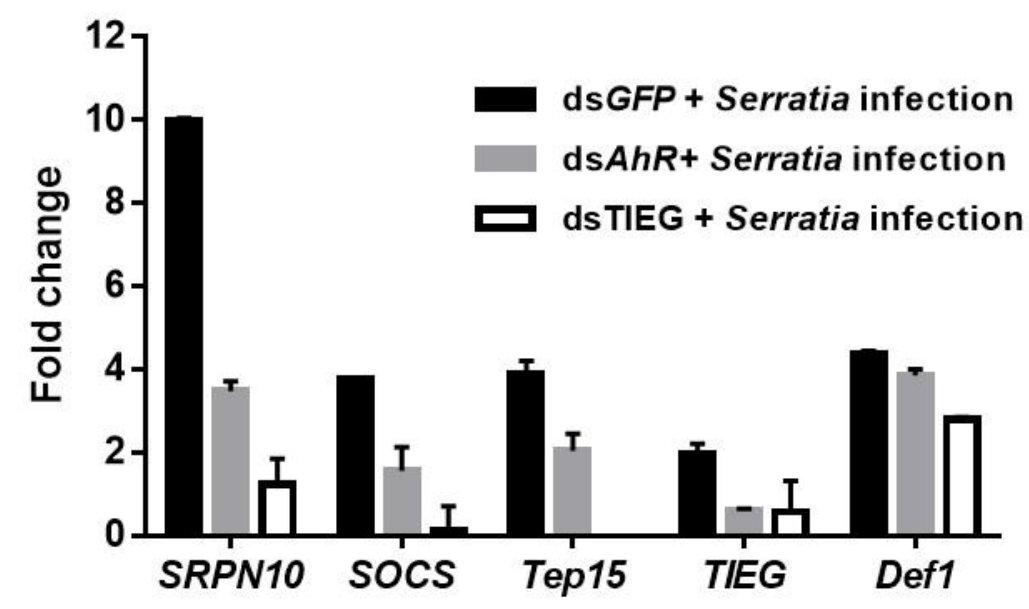

B.

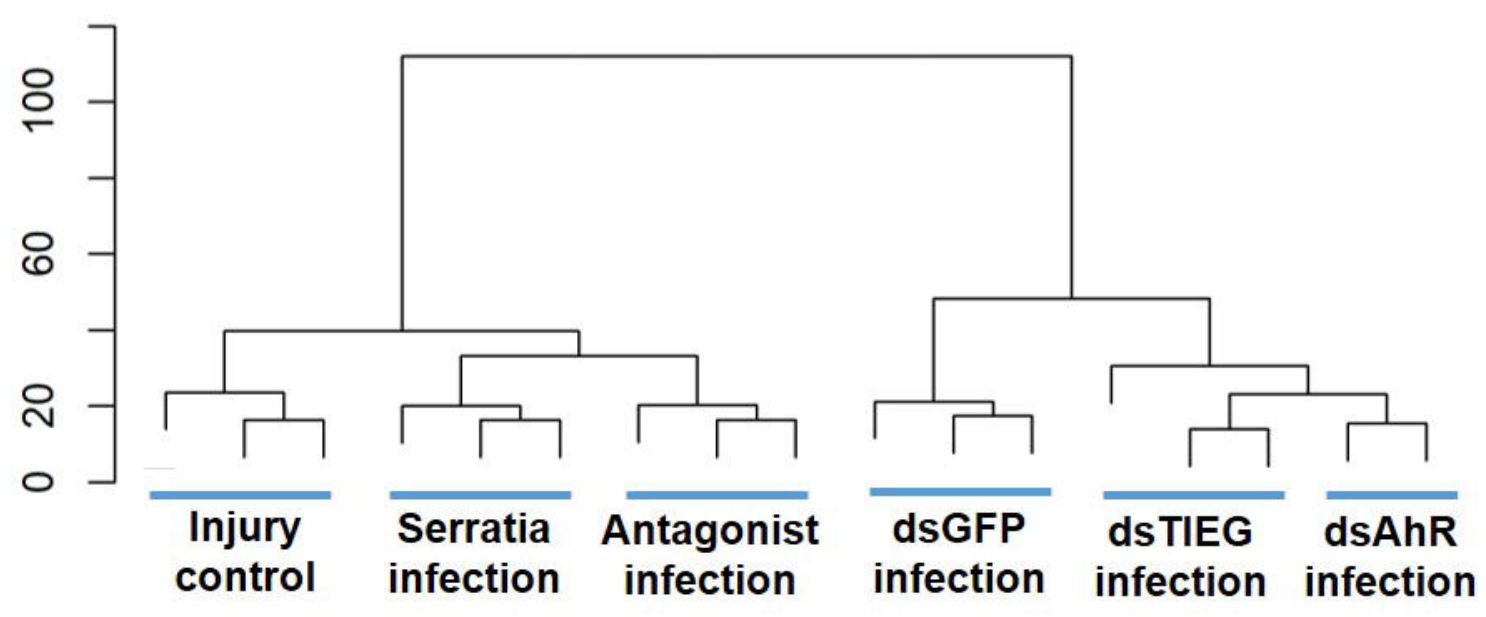

D.

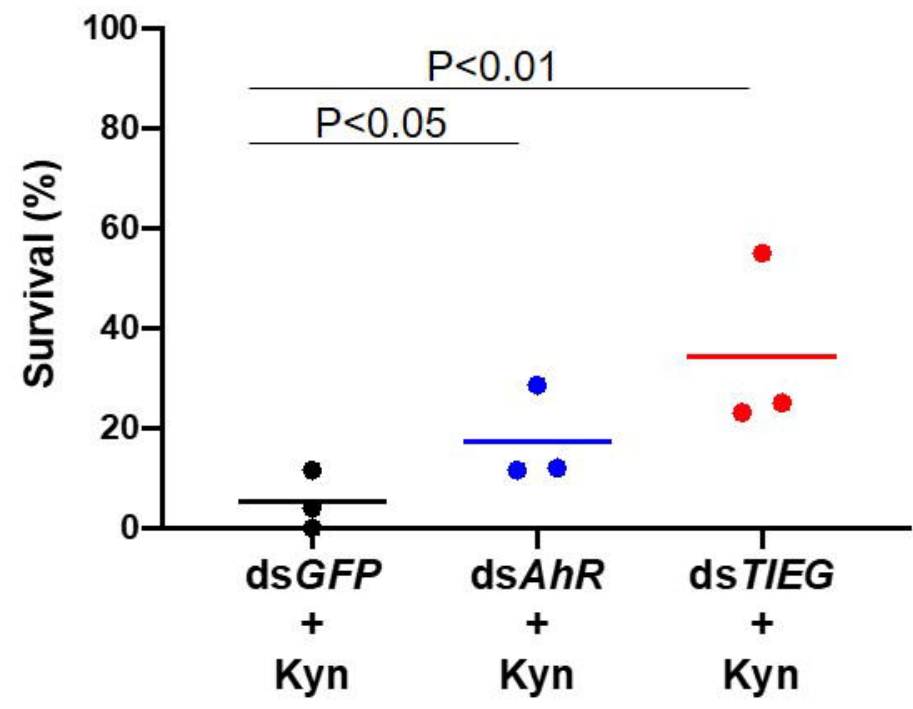

E. $\quad \frac{P<0.01}{P<0.01}$

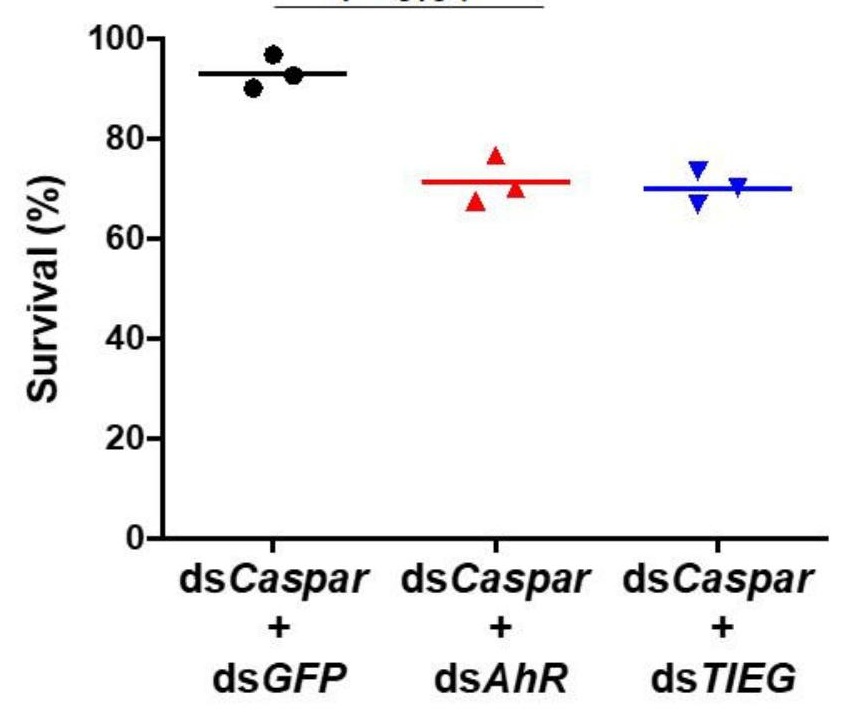




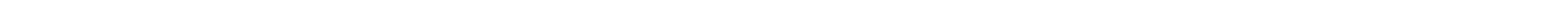


A.

73 Anopheles gambiae (Ag) 100 Aedes aegypti (Aa)

- Drosophila melanogaster (Dm)

Caenorhabditis elegans (Ce)

\begin{tabular}{|c|c|}
\hline & Danio rerio (Dr) \\
\hline 100 & - Homo sapiens (Hs) \\
\hline$\stackrel{\longmapsto}{\longmapsto .20}$ & - Mus musculus (Mm) \\
\hline
\end{tabular}

Ag v. Dm

Ag v. Dr

Ag v. Ce

Ag v. Hs
Domain protein sequence identity (\%)

\begin{tabular}{ccc}
$\begin{array}{c}\text { DNA } \\
\text { binding } \\
\text { bHLH }\end{array}$ & $\begin{array}{c}\text { Dimerization } \\
\text { PAS A }\end{array}$ & $\begin{array}{c}\text { Ligand } \\
\text { binding } \\
\text { PAS B }\end{array}$ \\
\hline 98 & 91 & 88 \\
69 & 49 & 44 \\
58 & 53 & 39 \\
72 & 59 & 44 \\
\hline
\end{tabular}

c.

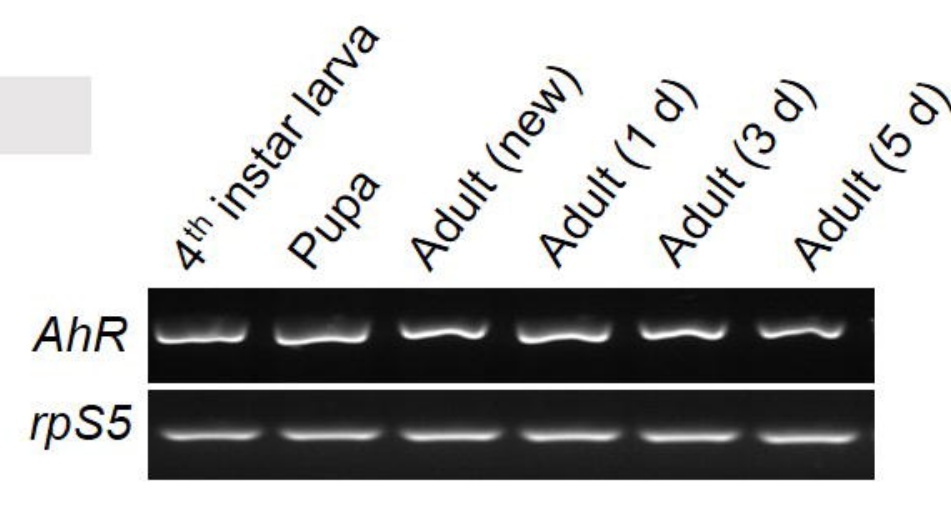




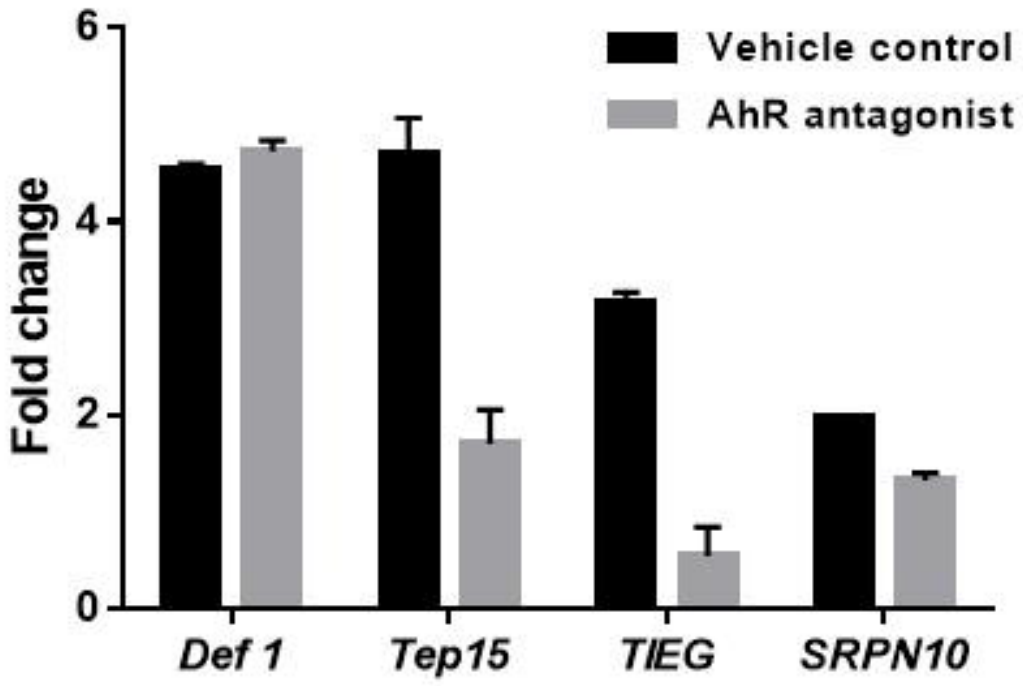


